\title{
The RNA-binding protein RGGA is a sugar- responsive cofactor of the 5'-3' exonuclease XRN4 that post-transcriptionally meditates plant growth
}

Hongmei Liu ( $\sim$ hmliu@sdau.edu.cn )

State Key Laboratory of Crop Biology, College of Life Sciences, Shandong Agricultural University https://orcid.org/0000-0002-1818-8334

$\mathrm{Ru} \mathrm{Yu}$

State Key Laboratory of Crop Biology, College of Life Sciences, Shandong Agricultural University Hong Zhang

State Key Laboratory of Crop Biology, College of Life Sciences, Shandong Agricultural University

Xinlei Gao

School of Life Sciences, University of Science and Technology of China

Changxiang Zhu

State Key Laboratory of Crop Biology, College of Life Sciences, Shandong Agricultural University

Changai Wu

Shandong Agricultural University

\section{Liwei Geng}

State Key Laboratory of Crop Biology, College of Life Sciences, Shandong Agricultural University Jinwen Du

State Key Laboratory of Crop Biology, College of Life Sciences, Shandong Agricultural University Haoran Ma

State Key Laboratory of Crop Biology, College of Life Sciences, Shandong Agricultural University Yunzhi Song

State Key Laboratory of Crop Biology, College of Life Sciences, Shandong Agricultural University Shumei Zhou

State Key Laboratory of Crop Biology, College of Life Sciences, Shandong Agricultural University

\section{Article}

Keywords: RGGA, sugar-regulated gene expression, AtXRN4

Posted Date: January 7th, 2021

DOl: https://doi.org/10.21203/rs.3.rs-131180/v1 
License: (c) (i) This work is licensed under a Creative Commons Attribution 4.0 International License. Read Full License 


\section{Abstract}

Sugar-regulated gene expression is a ubiquitous mechanism for carbohydrate allocation and utilization by keeping a balance among source and sink organs in plants. Previous studies have shown that excess sugar represses the expression of the genes implicated in photosynthesis and sugar metabolism. However, the mechanism is still largely unknown. Here, we found that the mutant of Arabidopsis RGGA, RGG repeats RNA-binding protein A coding gene, grew faster than wild type (Col-0) in MS medium. In rgga, mRNA half-live of the genes related to sucrose transport and metabolism, chlorophyll synthesis, root development as well as certain transcription factors was obviously longer than those of Col-0. Further study revealed that AtRGGA could interact with 5'-3' exonuclease AtXRN4, and guide it to the target mRNAs for their degradation. When AtRGGA is absent or its interaction domain is deleted, AtXRN4 self can't recognize the target mRNAs, which leads to a dramatically increase in transcript levels of the above gene subsets, and thus promotes the growth of Arabidopsis with exogenous sucrose supply. And only 5day sucrose supply could trigger the vigorous growth of rgga. These findings suggest that the regulation of mRNA stability mediated by RGGA plays a critical role in sugar suppression, and implicates a possibility to unlock the growth potential by modulating sugar utilization at post-transcriptional level in plants.

\section{Introduction}

In plants, sugars function as metabolic resources and structural constituent, and also function as signals that trigger gene expression changes associated with plant growth and development (Eveland and Jackson, 2012; Ruan, 2014). Indeed, we know that sugars are components of complex and interconnected signaling networks that allow plants to control cell division, growth, differentiation, and programmed cell death, including in responses to metabolic and environmental cues (Ho et al., 2001; Koch, 1996). The differential availability of soluble sugars is known to induce or repress the expression of a large variety of genes. The nature of sugar-mediated changes in gene expression is unique in plants, as these autotrophic organisms because their synthesis and allocation of carbohydrates can be modulated through photosynthesis/utilization balance (Koch, 1996). That is, plants can be considered as carbon heterotrophs at some stages during their life cycle, and some of their non-green organs like roots, stems, and flowers consistently function as sinks for carbon.

The photosynthetic and sugar metabolism genes have early been demonstrated to be repressed by photosynthetic product sucrose and glucose. That is known as sugar repression or sugar catabolite repression. And most studies on the mechanisms of sugar repression in plants have emphasized regulation at the transcriptional level (Chan et al., 1994; Graham et al., 1994; Sheen, 1990). Yet there is increasing awareness that sugar repression involves both transcriptional and mRNA stability related regulatory mechanisms. For example, three essential motifs in the promoter of the starch metabolism aamylase gene are responsible for its strong transcription, and the $3^{\prime}$-untranslated region (3'-UTR) of aamylase and its subdomains function independently as sugar-dependent mRNA stability determinants (Chan and Yu, 1998a, b; Lu et al., 1998; Sheu et al., 1996). 
Regulated stability $v s$. decay of mRNA transcript is now understood as a fundamental aspect for controlling eukaryotic gene expression. The 5'-3' and 3'-5' mRNA decay pathways were first identified in yeast and are now known to be conserved in multicellular eukaryotes (Chiba and Green, 2009; Mitchell and Tollervey, 2000). Highly unstable mRNA species in the cytoplasm are typically degraded through the 5'-3' mRNA decay pathway, which occurs in cytoplasmic foci called processing bodies (P-body) (Xu and Chua, 2011), whereas stable mRNAs are often degraded through the $3^{\prime}-5^{\prime}$ mRNA decay pathway occurs in both cytoplasmic and nuclear RNA exosomes (Puno et al., 2019; Song et al., 2013). The 5'-3' mRNA decay pathway is initiated upon the deadenylation and subsequent decapping of transcripts, which is catalyzed by the conserved eukaryotic decapping complex VARICOSE (VCS)-DCP1-DCP2; the decapped mRNAs are subsequently digested by 5' to 3' exoribonucleases (Rymarquis et al., 2011; Xu et al., 2006). Arabidopsis XRN4 is the functional homolog of yeast XRN1 and is known to degrade mRNAs and 3 ' cleavage products generated by the microRNA (miRNA) pathway (Rymarquis et al., 2011; Souret et al., 2004). Mutant analysis of Arabidopsis P-body components including DCP1, DCP2, VCS, and the exoribonuclease XRN4 have implicated 5'-3' mRNA decay was involved in postembryonic development, seed germination, thermal stress, as well as pathogen response (Basbouss-Serhal et al., 2017; Merret et al., 2013; Nguyen et al., 2015; Xu et al., 2006; Zhang et al., 2020).

Elevated sugar levels in plant cells have been shown to suppress the expression of a set of genes known to function in photosynthesis, reserve mobilization, and the export of sugars (Sheen, 1994; Thomas and Rodriguez, 1994; Yu, 1999). However, how this set of genes are coordinately regulated by sugar is still unclear. Here, in a screen for sucrose-responsive genes, we found that rgga, a mutant of Arabidopsis glycine-rich RNA binding protein, grew obviously faster in sucrose containing medium than wild type plants. We found that AtRGGA guides the 5'-3' exonuclease AtXRN4 to a subset of sucrose-responserelated $m R N A s$ for selective degradation. These transcripts are of genes with diverse functions including sugar carbohydrate metabolism, chlorophyll synthesis, and root development, and all detected mRNAs have common three motifs (UCUCWCUYUCDC, AGGAARRGRAA, YUUCUUCHCCU) in their $5^{\prime}$ UTRs and start codon near regions. When AtRGGA is absent, these mRNAs accumulate and trigger the vigorous long-term growth phenotypes we observed. Beyond demonstrating how RNA-binding proteins can act as cofactors to direct specific degradation via the 5'-3' mRNA decay pathway, and given that RGGA orthologs are widely present in both eudicots and monocots, our work suggests that combining disruption of RGGA function with sugar supplementation during planting represents a promising strategy for increasing the productivity of diverse crop species.

\section{Results}

\section{Exogenous sugar supplementation causes Arabidopsis rgga mutant plants to grow obviously faster than WT (Col-0) plants.}

We conducted a screen for sucrose responsive genes using a series of Arabidopsis mutant lines grown in MS medium supplemented with sucrose. rgga (SALK_143514), a deletion mutant of AtRGGA with a single T-DNA insertion located in its second exon, grew obviously faster than Col-0 in 2-3\% (w/v) sucrose MS 
medium (Fig. 1a). AtRGGA encodes a glycine-rich RNA binding protein (RBP), and its expression is induced by sucrose (Fig. 1b,c). AtRGGA is widely conserved in monocots and eudicots (Supplementary Fig. 1). After 2 weeks growth on MS media with $2 \%$ sucrose or then transported to vermiculite for additional 2 weeks growth, the biomass of rgga seedlings was significantly elevated compared to Col-0 seedlings (Fig. 1d,e).

To investigate whether the growth phenotypes result from sucrose specifically or sugar supplementation generally, rgga and Col-0 plants were grown in media supplemented with diverse sugars ( $2 \% \mathrm{w} / \mathrm{v})$, including three pentoses (ribose, xylose, and arabinose), three hexoses (glucose, mannose, and fructose), two disaccharides (sucrose and maltose), and one polysaccharide (soluble starch). Besides sucrose, glucose, mannose, fructose, and maltose also caused the significant rapid growth phenotypes for rgga seedlings (Fig. 1f). qRT-PCR analysis showed that the expression of AtRGGA was induced by all of the sugars used in this study (Supplementary Fig. 2).

We also conducted experiments in which seedlings were transplanted into vermiculite and allowed to developed through their reproductive growth stage without further exogenous sucrose supply. These experiments revealed that the growth advantage we observed for $2 \%$ sucrose supplemented rgga seedlings actually persisted throughout the whole Arabidopsis life cycle (Fig. 1g). The grain yield of rgga plants was significantly higher than Col-0 plants (Fig. 1h-j), and rgga seeds were larger in size and deeper in color than Col-0 seeds (Fig. 1k). Taken together, these results support that both the vegetative growth and reproductive growth capacities of Arabidopsis plants lacking the glycine-rich RNA binding protein AtRGGA can be increased beyond the capacities of WT plants ("exuberant growth") via supplementation with selected exogenous sugars during the seedling stage.

\section{Application of exogenous sucrose to rgga plants promotes root elongation and chlorophyll synthesis and increases the activity of sugar metabolism enzymes.}

To determine the minimum required time to induce the exuberant growth of rgga plants, we compared the growth of rgga and Col-0 plants that were initially grown in $2 \%$ sucrose MS for 2, 4, 5, or $6 \mathrm{~d}$ and then transferred to vermiculite and grown until they were assessed at day 28. No exuberant growth phenotypes were observed for the plants transplanted after 2 or 4 days of sucrose supplementation, but these phenotypes were evident for the plants transplanted after 5 or more days of supplementation. (Fig. 2a). We also conducted experiments further exploring the age window for rgga sensitivity to growth promoting effects from exogenous sucrose and detected exuberant growth phenotypes for rgga plants that were initially grown in sucrose free MS (for up to $8 \mathrm{~d}$ ) before being transplanted to $2 \%$ sucrose MS for continued growth (Supplementary Fig. 3). Thus, rgga plants are responsive to exogenous sucrose within a relatively large time window. However, it should be noted that the earlier and the longer the duration of the sucrose supplementation, the greater the extent of the detected growth differences between rgga and Col-0 plants.

To better understand the effects of AtRGGA mutation on growth and development, the roots of Col-0 and rgga which grown in $2 \%$ sucrose MS were observed. The root length of rgga is much longer than Col- 0 , 
with especially evident increases for the $6 \mathrm{~d}$ and $14 \mathrm{~d}$ supplementation samples. Further, we observed the presence of lateral roots in $14 \mathrm{~d}$ rgga, but not in $14 \mathrm{~d}$ Col-0 seedlings (Fig. 2b). Quantitative analysis showed that the $6 \mathrm{~d}$ and $14 \mathrm{~d}$ supplementation rgga seedlings had significant increases in both root length (Fig. 2C) and root meristem width (Supplementary Fig. 4a) compared to Col-0 seedlings.

Observation of the roots of wox5::GFP,rgga seedlings-in which quiescent center cells are marked by GFP -showed that the number and layer number of root apical meristem cells of wox5::GFP, rgga seedlings were obviously increased compared to wox5::GFP seedlings (Supplementary Fig. 4b). These results indicate AtRGGA deletion also enhances the root development of Arabidopsis plants given an exogenous sucrose supply.

In addition to exuberant growth, the rgga seedlings also had obviously greener leaves compared with Col0 . Chlorophyll content analysis showed that day 28 rgga plants with $\geq 5 \mathrm{~d}$ sucrose supplementation had significantly higher chlorophyll levels than similarly treated Col-0 plants (Fig. 2d). Sugar metabolism is closely related to plant growth and development, and we analyzed the activities of several enzymes involved in sugar metabolism in the plants that were initially exposed to exogenous sucrose and then transplanted to vermiculite. Classic in vitro assays with total protein extracts from leaves showed that at day 28, the activities detected for neutral invertase ( $\mathrm{NI}$ ), acid invertase (Al), and hexokinase (HK) were all significantly higher in extracts from the $\geq 5 \mathrm{~d}$ sucrose supplemented rgga plants than in Col-0 or $<5 \mathrm{~d}$ supplemented rgga plants (Fig. 2e-g). Note that these observations for the increased activity of sugar metabolism enzymes are consistent with the exuberant growth phenotype data for the corresponding plants (Fig. 2a). These results indicate that AtRGGA deletion promotes chlorophyll levels and the activity of sugar metabolism enzymes in Arabidopsis plants given exogenous sucrose.

\section{rgga plants given exogenous sucrose have strongly elevated expression of genes related to carbohydrate metabolism, chlorophyll synthesis, and root development.}

To investigate how AtRGGA functions in growth, we conducted transcriptome analysis of Col-0 and rgga seedlings that were cultivated for $6 \mathrm{~d}$ or $14 \mathrm{~d}$ in $2 \%$ sucrose MS. Notably, there were relatively more upregulated rgga DEGs at the $14 \mathrm{~d}$ supplementation time point as opposed to that of Col-0, although there were many more down-regulated genes among 987 at $6 \mathrm{~d}$ and 110 at $14 \mathrm{~d}$ differentially expressed genes (DEGs) in rgga seedlings compared with Col-0 (Fig. 3a). A GO analysis comparing the DEGs for the $6 \mathrm{~d}$ rgga and Col-0 samples indicated enrichment in among the up-regulated rgga DEGs for annotations related to root meristem growth, transcription regulation, ubiquitin protein ligase activity regulation and carbohydrate transport (Supplementary Fig. 5). The expression levels of 19 transcript factors (TFs) were increased in rgga, among which 7 have been previously implicated in growth regulation including GRF1, SPL2, SPL10, AN3, SAP, PLT1, and PLT2 (Galinha et al., 2007; Kim et al., 2003; Shikata et al., 2009; Shishkova et al., 2008; Vercruyssen et al., 2014; Wang et al., 2016) (Fig. 3b).

The rgga root growth phenotypes focused our attention on the DEGs that have been previously reported to exhibit root-specific expression profiles. There were 25 such genes among the DEGs (Supplementary Fig. 6), including $P L T 1$ and $P L T 2$, two master transcription factors known to promote root development 
(Galinha et al., 2007). Recalling the increased chlorophyll levels in rgga seedlings, we also analyzed the differential expression of genes with functional annotations related to chlorophyll synthesis and photosynthesis. Few DEGs in rgga compared to Col-0 with such annotations including HEMF2, HEMA2, HEME2, and PsbP-2 were identified.

Beyond sucrose metabolism, chlorophyll content, and root development, and recalling the exuberant growth phenotypes in the sucrose-supplemented rgga plants, it was highly interesting that we found that 33 DEGs with functional annotations related to plant cell wall organization were down-regulated in rgga (Supplementary Tab. 1). As one example, the function of $P R X 71$ is to strengthen cell wall and thus limit cell expansion during normal growth and in response to cell wall damage (Raggi et al., 2015). Further investigation reveals that 22/33 genes involved in cell wall organization are enriched in root, and 5/22 have proven to be involved in root elongation (PRX44, PRX57, EXT6, EXT13, EXPA7) (Kwon et al., 2015; Lan et al., 2013; Petricka et al., 2012). This suggest that the root growth of rgga plants might be affected under this condition.

To validate both the previous phenotypes and expression trends from our transcriptome analysis, we used qPCR to assess the expression levels of genes including $N I, A l, H K$, sucrose transporter genes, chlorophyll synthesis related genes, root development related genes, growth regulation TFs and plant cell wall organization genes in rgga and Col-0 seedlings grown on $2 \%$ sucrose or sucrose-free MS for $14 \mathrm{~d}$. In the absence of sucrose supplementation, we detected no significant differences in expression levels of the detected genes between rgga and Col-0 seedlings. Upon exogenous sucrose supply, we found significantly elevated expression levels in sucrose-supplemented rgga seedlings for genes including the NI genes (INVC and INVD), Al genes (CWIN2, CWIN4, and CWIN5), HK genes (FLN2, HXK3, HKL 1), sucrose transporter genes (SUC3, SUC4, SUC7, SGB1), chlorophyll synthesis related genes (PORB, PORC, CAO), and growth regulation TFs ( $P L T 1, P L T 2, G R F 1, S P L 2, S P L 10, A N 3$, and SAP) compared to similarly treated Col0 plants among the detected genes (Fig. 3c,d and Supplementary Fig. 7). Notably, the expression of the above genes was induced by $2 \%$ sucrose both in Col-0 and rgga plants (Fig. $3 \mathrm{c}, \mathrm{d}$ ). And qPCR analysis showed that the cell wall organization genes PRX44, PRX57, EXT6, EXT13, EXPA7, PRX71 were induced by sucrose in Col-0 plants and down-regulated in rgga plants grown on $2 \%$ sucrose MS (Supplementary Fig. 8).

\section{AtRGGA mutation increases the mRNA stability of carbon metabolism chlorophyll synthesis, and root development related genes.}

As an RNA binding protein, AtRGGA may affect the expression of its apparent target genes by affecting the stability of their transcripts. Pursuing this speculation, we carried out mRNA stability assays for DEGs in rgga and Col-0. Two-week-old seedlings growing in $2 \%$ sucrose MS were treated with the transcription inhibitor Actinomycin D (Act D), and then the mRNA stability of INVC, INVD, CWIN2, CWIN4, HXK3, FLN2, SUC3, SGB1, PORB, CAO, PLT1, and PLT2 were examined across a time course of one hour. The remaining transcripts of these genes of Col-0 decreased faster than that of rgga as time went on (Fig. 4a). Additionally, the mRNAs of four TFs of rgga which involved in growth regulation were proved to be more 
stability than that of Col-0 (Supplementary Fig. 9). These results suggest AtRGGA indeed reduces the mRNA stability of the detected genes.

To further explore how AtRGGA influences the mRNA stability of the carbon metabolism, chlorophyll synthesis, and root development related genes, RNA immunoprecipitation (RIP) combined with qPCR was conducted using GFP and AtRGGA-GFP transgenic plants grown on $2 \%$ sucrose MS for $14 \mathrm{~d}$. The results showed that AtRGGA specifically bound to the 5'-UTR and the downstream regions close to the start codon of INVC, CWIN2, HXK3, SUC3, PORB, and PLT1 transcripts (Fig. 4b and Supplemental Fig. 10). We also searched the motifs overrepresented in the 5 ' terminus for 16 transcripts (AtRGGA binding regions identified by RIP for 6 transcripts and 1-800bp for 10 transcripts of which stability detected) specifically more abundant and stable in rgga mutant using MEME (Version 5.1.1) (Bailey and Elkan, 1994), and three common motifs (UCUCWCUYUCDC, AGGAARRGRAA, YUUCUUCHCCU) were invariably present in these transcripts (E values=7.2e-007, 1.2e-002, 4.7e-002 respectively) (Fig. 4c and Supplemental Fig. 11).

Note that we transformed a combined $\triangle R G G 1 \& 2 /$ rgga deletion variant of AtRGGA into the rgga background Arabidopsis and grew these plants in 2\% sucrose MS for 14 days, the growth phenotypes of $\triangle R G G 1 \& 2 /$ rgga plants were similar to rgga (Fig. 5f). This is unsurprising, given the roles of the RGG1 and RGG2 domains in RNA binding (Landsberger et al., 2002). The mRNA levels of INVC, CWIN2, HXK3, SUC3,

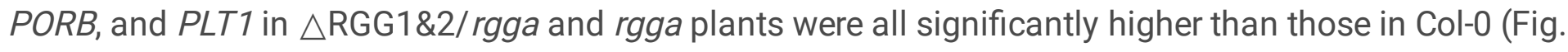
$4 \mathrm{~d})$. These results indicate that the sucrose-dependent, suppressive regulation of AtRGGA on growth depends on its binding with mRNA targets.

\section{AtRGGA interacts with the 5'-3'exonuclease AtXRN4.}

RGGA binding to the $5^{\prime}$ terminus of mRNAs raises the possibility that RGGA may regulate mRNA stability through a 5' to 3' mRNA decay pathway (Braun et al., 2016; Merret et al., 2013). We therefore used Y2H assays to explore interactions between AtRGGA and known proteins involved in P-body formation including Arabidopsis DCP1, DCP2, VCS and exoribonuclease XRN4. Strong activation of the reporter gene was detected in yeast cells co-expressing AtRGGA-BD and AtXRN4-AD, indicating that AtRGGA can physically interact with AtXRN4 (Fig. 5a). AtXRN4 was shown to interact with AtDCP1 and AtDCP2 as previously reported, but AtRGGA did not interact with them (Supplemental Fig. 12). Subsequent bimolecular fluorescence complementation (BiFC) assays and co-immunoprecipitation (Co-IP) further supported the interaction between AtRGGA and AtXRN4 in plant (Fig. 5b,c).

Primary structure analysis using a web-based tool SMART as well as the structure reported previously (Landsberger et al., 2002), five functional domains in AtRGGA, including Stm1, HABP4-PAI-RBP1, two RGG boxes, and two resemble bipartite nuclear localization signals (NLSs) were predicted (Fig. 5d). Notably, although having two presumed NLSs, AtRGGA is located perinuclear region of the cytoplasm rather than nucleus according previous study (Ambrosone et al., 2015) and our observations (Supplemental Fig. 13). And the perinuclear localization of AtRGGA can also be seen in our BiFC results (Fig. 5b). Co-IP assays with AtRGGA domains deletion variants (transiently expressed in $N$. benthamiana leaves) showed that NLS1 (EKKKALQSLTTSER KV) was essential for the AtRGGA-AtXRN4 interaction. Deletions of Stm1, the 
two RGG boxes, HABP4-PAI-RBP1, and NLS2 did not influence the interaction (Fig. 5e). We stably transformed the AtRGGA NLS1 deletion variant into the rgga background, and cultivated these deletion variant lines in $2 \%$ sucrose MS for $14 \mathrm{~d}$. The $\triangle \mathrm{NLS} 1 /$ rgga line grew significantly faster than Col-0, and exhibited the same growth phenotypes as the rgga plants (Fig. 5f). The mRNA levels of INVC, CWIN2, $H X K 3$, SUC3, PORB, and PLT1 in $\triangle \mathrm{NLS1}$ /rgga and rgga plants were all significantly higher than those in Col-0 (Fig. 5g). These results confirm that AtRGGA's NLS1 domain is essential for its sucrose-dependent suppression of seedling growth.

\section{The exoribonuclease XRN4 degrades RGGA-bound mRNAs.}

Given our identification of AtRGGA target mRNAs and their common motifs located in the $5^{\prime}$ UTRs and their adjacent downstream regions, and considering the known mRNA digestion role of XRN4 in the $5^{\prime}$ to 3' mRNA decay pathway, it seems plausible that the AtRGGA-AtXRN4 interaction may facilitate the selective degradation of AtRGGA-targeted mRNAs. Pursuing this, we generated tagged materials and conducted RIP assays using HA and GFP antibodies; specifically, we generated AtXRN4-HA/rgga, AtRGGA-GFP/xrn4, and AtXRN4-HA lines, and also used the aforementioned AtRGGA-GFP line. Seedlings of these lines were grown in $2 \%$ sucrose MS for $14 \mathrm{~d}$ and assessed with RIP assays. Fragment enrichment in RIP products was detected by qPCR, with segmented primer pairs for AtRGGA target mRNAs including INVC, CWIN2, HXK3, SUC3, PORB, and PLT1 (Fig. 6a and Supplemental Fig. 14).

AtXRN4 did not bind to these AtRGGA target mRNAs in the absence of RGGA. Further, AtXRN4 was not required for AtRGGA binding to its target mRNAs. Additionally, these assays showed that, for all of its target mRNAs, AtRGGA bound to a 5'UTR and its adjacent region that contained the common motifs. Viewed alongside our AtRGGA-AtXRN4 interaction data, these results support a model wherein AtRGGA binds its targets in their 5'UTRs and 5'UTR near regions, and the exoribonuclease AtXRN4 can then recognize these AtRGGA-bound mRNA substrates for degradation. Pursuing this, we tested the growth of rgga, xrn4-5, AtXRN4-HA/rgga, AtRGGA-GFP/xrn4-5 and rgga,xrn4-5 plants cultured in $2 \%$ sucrose MS for $14 \mathrm{~d}$. The rosettes size of AtXRN4-HA/rgga, AtRGGA-GFP/xrn4-5 and rgga,xrn4-5 was not different to that of rgga, but obviously larger than that of Col-0 (Fig. 6b).

The deletion of NLS1 which destroying the interaction of AtRGGA and AtXRN4 increased the accumulation of carbon metabolism, chlorophyll synthesis, and root development mRNAs (Fig. 5e), and AtXRN4 did not bind to these mRNAs in the absence of AtRGGA (Fig. 6a). It suggested that AtRGGA is probably responsible for the specificity of the mRNA degradation conducted by AtXRN4. Given the RGGA target mRNAs we identified, and considering the known digestion role of XRN4 in the 5' to 3' mRNA decay pathway, it seems plausible that the AtRGGA-AtXRN4 interaction may facilitate the selective degradation of RGGA-targeted mRNAs.

\section{Discussion}

As the main transport form of photosynthate, sucrose participates in the balance regulation of source and sink to meet the needs of growth and stress resistance in plants (Chan and Yu, 1998a; Ruan, 2014). 
Studies have established that elevated sucrose decreases the expression of genes that function in photosynthesis, sugar catabolism, and sugar transport (Koch, 1996; Paul and Pellny, 2003; Sheen, 1994). However, the mechanism through which these diverse genes are rapidly and simultaneously repressed remains unclear. In the present study, we show that the RNA-binding protein AtRGGA functions to allow the 5'-3' exonuclease AtXRN4 to recognize a subset of sucrose transport and metabolism, chlorophyll synthesis, and root development related mRNA transcripts for degradation. When AtRGGA or AtXRN4 is absent, the accumulation of these mRNAs increases substantially, thus enabling the observed rapid growth of mutant plants given an exogenous sucrose supply (Fig. 6c).

Among the three XRN enzymes of Arabidopsis, AtXRN4 is the only one that functions in cytoplasmic 5'-3' mRNA degradation (Kastenmayer and Green, 2000). AtXRN4 has been shown to promote the decay of some but not all mRNAs, including transcripts that code for proteins of ethylene signaling, light signaling, seed germination, and ABA/GA metabolism, as well as heat stress and nitrogen stress responses (Basbouss-Serhal et al., 2017; Estavillo et al., 2011; Merret et al., 2013; Nagarajan et al., 2019; Nguyen et al., 2015; Olmedo et al., 2006; Rymarquis et al., 2011; Wawer et al., 2018). Our results demonstrate that AtXRN4 recognizes subsets of mRNAs related to sucrose transport and metabolism, chlorophyll synthesis, and root development, and we show that this specific recognition is mediated by the RNAbinding protein AtRGGA. This echoes previous demonstrations that AtXRN4's recognition of a subset of mRNAs under thermal stress requires help from the RNA-binding protein AtLARP1 (Merret et al., 2013). Notably, the yeast RNA-binding protein VTS1 was shown to function in glucose-induced mRNA decay (Braun et al., 2016). Viewed together, these results clearly indicate that the specificity of XRN-mediated RNA decay is mediated by particular RNA-binding cofactor proteins. Thus, the further identification and functional characterization of such cofactors will almost certainly deepen our understanding of specific mRNA degradation programs triggered by particular conditions, including environmental stresses, nutrients, and specific developmental stages.

Biochemically, we discovered that AtRGGA guides AtXRN4 to interact with target mRNAs via binding to their $5^{\prime}$ UTRs and 5' UTR downstream regions. This binding pattern matches our understanding of AtXRN4 as a 5'-3' exonuclease; however, it is not yet clear how the AtXRN4 cofactor AtRGGA recognizes its own target transcripts. Previous works has demonstrated specific mRNA sequences especially some hexamer motifs from select functional categories were attractive targets for XRN4-mediated decay (Basbouss-Serhal et al., 2017; Rymarquis et al., 2011). In addition, the factors known to influence AtXRN4's binding with degradation substrate mRNAs also include the GC content of 5 ' UTR, the poly (A) tail at the $3^{\prime}$ end, as well as $\mathrm{N}^{6}$-methyladenosine $\left(\mathrm{m}^{6} \mathrm{~A}\right)$ modification of transcripts (Basbouss-Serhal et al., 2017). We discovered here that all of the transcripts whose stability increase in rgga contain three motifs in their $5^{\prime}$ UTRs and adjacent regions, suggesting that AtRGGA might recognize its target mRNAs through these common motifs. It should be interesting to elucidate precisely which structural and biochemical factor(s) of these common motifs mediate AtRGGA's recognition of its target mRNAs.

High yield is obviously a major target of crop breeding efforts, and we know that yield is strongly impacted by source-sink balance for sugars (Smith et al., 2018). Given the strong developmental impacts 
of sugar repression in plants, simply increasing source strength does not work particularly well to obtain yield improvements (Li et al., 2018; Rodrigues et al., 2019). In the present study, both rgga and xrn4-5 plants showed rapid growth phenotypes upon exogenous sucrose supply. Compared with $X R N 4$, which is widely involved in global signal responses in plant cells, RGGA may be a more suitable candidate gene for growth improvement manipulation. For example, Arabidopsis mutants of XRN4 could not survive a long period of moderately high temperature, whereas mutation of LARP1, a heat-specific cofactor of AtXRN4, did not cause a similar extent of heat-induced damage (Merret et al., 2013). RGGA specifically interacts with (and thus regulates) sugar metabolism, chlorophyll synthesis, and root development related genes, making it an excellent candidate for yield improvement. Recall our finding that sucrose supply did not trigger faster growth of rgga over Col-0 when the treated seedlings were younger than $5 \mathrm{~d}$. We suspect this is because the heterotrophy-autotrophy transition-which enables Arabidopsis seedlings to gain their photosynthetic competence (Chen and Thelen, 2010)-occurs at this time. The growth effects of the rgga mutation seem to follow the "Matthew effect"; that is, early exogenous supplementation with sugar promotes root development and chlorophyll synthesis, thereby greatly improving nutrient absorption and photosynthetic efficiency, thus increases the overall biomass of Arabidopsis.

Plant molecular physiology studies have shown that both transcriptional and post-transcriptional regulation mechanisms mediate sugar repression effects. Our results suggested that mRNA stability regulation even played a more crucial role in sugar repression given the coverage of the involved genes. Beyond the role we demonstrate for AtRGGA in sugar repression, it is known that both drought and salt stress induce the accumulation of the AtRGGA protein (Ambrosone et al., 2015). Thus, it seems plausible that the specific target mRNA binding and AtXRN4 cofactor model we illustrate here for Arabidopsis in the present study may help explain post-transcriptional mechanisms underlying plant responses to these major forms of environmental stress. Our results suggest that rgga does not obviously effect drought resistance compared to Col-0 when sucrose is supplied at an early growth stage. Additionally, it bears emphasis that salt and drought stress are not major limits to plant growth in many agricultural production regions. Thus, given the ubiquitous conservation of the RGGA gene among eudicots and monocots, it seems that combined manipulation of the RGGA gene alongside adjusted cultivation measures (i.e., sucrose supplementation during planting and photosynthesis improvement) could result in major gains in the productivity of many crop plants.

\section{Methods}

Plant materials. All the wild-type, mutant and transgenic Arabidopsis lines used throughout this study are Columbia (Col) ecotype. Seeds of rgga (SALK_143514), xrn4-5 (CS68822) are purchased from Arabidopsis Biological Resource Center. Both AtRGGA and AtXRN4 genes with corresponding tags are amplified from Col-0 cDNA and ligated into $\mathrm{pBI} 121$ and pBI121-GFP vectors through appropriate restriction sites. The 35S::AtRGGA, 35S::AtXRN4, 35S::AtRGGA-GFP, and 35S::AtXRN4-HA transgenic lines are obtained by floral dipping of Col-0. 35S::AtXRN4-HA/rgga and 35S::AtRGGA-GFP/xrn4 lines are obtained by floral dipping of rgga and $x \mathrm{rn} 4$ with corresponding structure-containing Agrobacterium, respectively. WOX5::GFP/rgga line was acquired by hybridizing WOX5::GFP and rgga. WUSCHEL- 
RELATED HOMEOBOX gene (WOX5) is specially expressed in the quiescent center of Arabidopsis root. The transgenic lines are identified by antibiotic screening and PCR amplification using specific primers (Supplemental Table 2). All plants are grown on MS medium or vermiculite under standard long-day condition ( $16 \mathrm{~h}$ of light, $8 \mathrm{~h}$ of dark) at $22^{\circ} \mathrm{C}$.

Growth phenotype analysis. Fresh weight of Col-0 and rgga plants which grown on $2 \%$ sucrose MS for 2 weeks or then transported to vermiculite for further 2 weeks growth is weighed. After drying in an oven at $80^{\circ} \mathrm{C}$ for 2 days, dry weight of the above seedlings is weighed. Three biological replicates are set up, and each replication including 50 plants. Other groups of Col-0 and rgga firstly grown on $2 \%$ sucrose MS for 2 weeks and then transported to vermiculite for further growth until they make seeds. And three sets of 100 seeds are weighed to obtain the data of hundred-grain weight. In addition, the number of seeds per plant of rgga and Col-0 is separately counted. It replicates for three times, and each replication includes 5 plants.

Chlorophyll content assays. $0.2 \mathrm{~g}$ leaves are soaked in $80 \%$ acetone solution for $48 \mathrm{~h}$, and then the absorbance values at $645 \mathrm{~nm}$ and $663 \mathrm{~nm}$ of the extract are measured by using a spectrophotometer. Three biological replicates are set up. According to the absorption value of chloroplast pigment and the Lambert-Beer law, the pigment content is calculated using the following formulas.

$C=20.3^{\star} A_{645 n m}+8.04^{\star} A_{663 n m}$

$\rho=C * V /(W * 1000)$

Where A indicates the absorption of the extract at the corresponding wavelength, $\rho$ is the chlorophyll content (mg・g-1); $C$ is the corresponding chlorophyll concentration in the acetone extract $(m g \cdot L-1) ; V$ is the solution volume $(\mathrm{mL}) ; \mathrm{W}$ is the sample fresh weight $(\mathrm{g})$.

Enzyme activity analysis. The activities of neutral invertase (NI), acid invertase (Al), and hexokinase (HK) of Col-0 and rgga which grown on $2 \%$ sucrose MS for a few days and then transported to vermiculite for further growth till $28 \mathrm{~d}$ age were assayed. $1 \mathrm{~g}$ leaf is ground with $3 \mathrm{~mL}$ extract buffer ( $\mathrm{PH} 7.5,0.05 \mathrm{M}$ phosphate buffer, $0.1 \mathrm{mM}$ EDTA, $1 \mathrm{mM}$ cysteine, and $10 \mathrm{mM} \mathrm{NaSO}_{3}$ ). Then the extract is centrifuged at $20,000 \mathrm{rpm}$ for $15 \mathrm{~min}$ at $4^{\circ} \mathrm{C}$. The supernatant liquid is regarded as crude enzyme. $\mathrm{Nl}$ activity is determined as following. $0.2 \mathrm{~mL}$ of crude enzyme is added into $1.2 \mathrm{~mL}$ of $0.2 \mathrm{M}$ phosphate buffer $(\mathrm{pH}=7.5)$ and $0.2 \mathrm{~mL}$ of $0.3 \mathrm{M}$ sucrose. The above solution added $2 \mathrm{~mL}$ of $1 \mathrm{M} \mathrm{NaOH}$ immediately is taken as the control. The above solutions are incubated at $35^{\circ} \mathrm{C}$ for $30 \mathrm{~min}$, then $2 \mathrm{~mL}$ of $1 \mathrm{M} \mathrm{NaOH}$ and 5 $\mathrm{mM}$ NDS are added. The mixtures are boiled in a water bath for $5 \mathrm{~min}$ and the absorbance is measured at $540 \mathrm{~nm}$. The enzyme activity is calculated through a standard curve and in $\mu \mathrm{mol}$ Suc/mg Pr.h. In the case of $\mathrm{Al}$ activity assay, $0.2 \mathrm{M}$ acetate buffer is used instead of $0.2 \mathrm{M}$ phosphate buffer (pH 7.5). And HK activity is analysed with Hexokinase assay kit (Cominbio). All measurements are replicated three times.

Transcriptome sequencing. Transcriptome sequencing for rgga and Col-0 whole plants grown on $2 \%$ sucrose MS for $6 \mathrm{~d}$ and $14 \mathrm{~d}$ respectively is carried out using Illumina HiSeqX10/HiSeq4000 platform. 
And three biologically replicated materials are provided.

qRT-PCR. Total RNA is extracted from leaves by using TRIzol Reagent (Invitrogen), treated with DNase区. Complementary DNA (CDNA) is synthesized from $1 \mu \mathrm{g}$ of total RNA by using FastKing gDNA dispelling RT SuperMix (Tiangen). Real-time PCR is performed with the LightCycler 480 SYBR Green I Master (Roche) in a $15 \mu \mathrm{L}$ reaction volume. As a reference gene, actin is amplified. The primers are presented in Supplemental Table 2. The instrument used for detection is LightCycler 96 (Roche). Results presented are means of three biological replicates.

RNA immunoprecipitation assays. $3 \mathrm{~g}$ whole plant of Arabidopsis is harvested into a centrifuge tube containing $37 \mathrm{~mL}$ of $1.0 \%$ formaldehyde and $2.5 \mathrm{~mL}$ of $2 \mathrm{M}$ glycine, and then infiltrated for $15 \mathrm{~min}$ in a desiccator attached to a vacuum pump. Then RNA samples are extracted and purified. qPCR is used to detect the expression level of mRNAs (Zhang et al., 2016).

mRNA stability analysis. The rgga and Col-0 seedlings grown in MS medium ( $2 \%$ sucrose) for $14 \mathrm{~d}$ are treated with actinomycin D (Act-D) and collected every 15 min across one hour. mRNAs of the treated plants are extracted and reverse-transcribed to CDNAs for qPCR assay. This experiment is replicated for three times. Primers are mentioned in Supplemental Table 2.

Apical structure observation. WOX5::GFP/rgga lines are obtained by hybridizing WOX5::GFP and rgga lines. The WOX5::GFP and WOX5: GFP/rgga seedlings grown in $2 \%$ sucrose MS medium for $4 \mathrm{~d}, 6 \mathrm{~d}$ and $14 \mathrm{~d}$, and the apical structures were observed using two-photon laser confocal microscope (TPLSCM).

Protein interaction analysis. $\mathrm{Y} 2 \mathrm{H}$ assays are carried out using the GAL4-based two-hybrid system as described in the Yeast maker Yeast Transformation System 2 User Manual (Clontech). The cDNAs of AtRGGA, AtRGGA deletion mutants and AtXRN4 are cloned into pGBKT7 and pGADT7 respectively. And the recombination plasmids are co-transformed into AH109, followed by incubation on the QDO/X-agal/AbA medium.

AtRGGA and AtXRN4 are cloned by Gateway technology to obtain BiFC test vectors AtRGGA-YFPC, AtRGGA-YFPN, AtXRN4-YFPC, AtXRN4-YFPN. And these recombination vectors are transformed to Agrobacterium GV3101 and used to carry out transient infiltration. About $1 \mathrm{~cm}^{2}$ infiltrated tobacco leaf at a distance of 2-3 mm from the pinhole is cut, placed flat on a glass slide, and then observed with TPLSCM for YFP fluorescent signals (Tang et al., 2020).

Co-IP assay is performed as described by Tang et al. (Tang et al., 2020) and the films are washed by TTBS (Tris Buffered Saline, with Tween-20, pH 8.0) and then photographed using Tanon-5500 (Tanon). Bound proteins are detected by immunoblotting using anti-His, anti-HA, anti-GFP (Beyotime) antibodies respectively.

\section{References}


Ambrosone, A., Batelli, G., Nurcato, R., Aurilia, V., Punzo, P., Bangarusamy, D.K., Ruberti, I., Sassi, M., Leone, A., Costa, A., et al. (2015). The Arabidopsis RNA-binding protein AtRGGA regulates tolerance to salt and drought stress. Plant physiology 168, 292-306.

Bailey, T.L., and Elkan, C. (1994). Fitting a mixture model by expectation maximization to discover motifs in biopolymers. Proceedings International Conference on Intelligent Systems for Molecular Biology 2, 2836.

Basbouss-Serhal, I., Pateyron, S., Cochet, F., Leymarie, J., and Bailly, C. (2017). 5' to 3' mRNA Decay Contributes to the Regulation of Arabidopsis Seed Germination by Dormancy. Plant physiology 173 , 1709-1723.

Braun, K.A., Dombek, K.M., and Young, E.T. (2016). Snf1-Dependent Transcription Confers GlucoseInduced Decay upon the mRNA Product. Molecular and cellular biology 36, 628-644.

Chan, M.T., Chao, Y.C., and Yu, S.M. (1994). Novel gene expression system for plant cells based on induction of alpha-amylase promoter by carbohydrate starvation. The Journal of biological chemistry $269,17635-17641$.

Chan, M.T., and Yu, S.M. (1998a). The 3' untranslated region of a rice alpha-amylase gene functions as a sugar-dependent mRNA stability determinant. Proceedings of the National Academy of Sciences of the United States of America 95, 6543-6547.

Chan, M.T., and Yu, S.M. (1998b). The 3' untranslated region of a rice alpha-amylase gene mediates sugar-dependent abundance of mRNA. The Plant journal : for cell and molecular biology 15, 685-695.

Chen, M., and Thelen, J.J. (2010). The Plastid Isoform of Triose Phosphate Isomerase Is Required for the Postgerminative Transition from Heterotrophic to Autotrophic Growth in Arabidopsis. The Plant cell 22, 77-90.

Chiba, Y., and Green, P.J. (2009). mRNA Degradation Machinery in Plants. Journal of Plant Biology 52, 114-124.

Estavillo, G.M., Crisp, P.A., Pornsiriwong, W., Wirtz, M., Collinge, D., Carrie, C., Giraud, E., Whelan, J., David, P., Javot, H., et al. (2011). Evidence for a SAL1-PAP chloroplast retrograde pathway that functions in drought and high light signaling in Arabidopsis. The Plant cell 23, 3992-4012.

Eveland, A.L., and Jackson, D.P. (2012). Sugars, signalling, and plant development. Journal of experimental botany 63, 3367-3377.

Galinha, C., Hofhuis, H., Luijten, M., Willemsen, V., Blilou, I., Heidstra, R., and Scheres, B. (2007). PLETHORA proteins as dose-dependent master regulators of Arabidopsis root development. Nature 449, 1053-1057. 
Graham, I.A., Denby, K.J., and Leaver, C.J. (1994). Carbon Catabolite Repression Regulates Glyoxylate Cycle Gene Expression in Cucumber. The Plant cell 6, 761-772.

Ho, S., Chao, Y., Tong, W., and Yu, S. (2001). Sugar coordinately and differentially regulates growth- and stress-related gene expression via a complex signal transduction network and multiple control mechanisms. Plant physiology $125,877-890$.

Kastenmayer, J.P., and Green, P.J. (2000). Novel features of the XRN-family in Arabidopsis: evidence that AtXRN4, one of several orthologs of nuclear Xrn2p/Rat1p, functions in the cytoplasm. Proceedings of the National Academy of Sciences of the United States of America 97, 13985-13990.

Kim, J.H., Choi, D., and Kende, H. (2003). The AtGRF family of putative transcription factors is involved in leaf and cotyledon growth in Arabidopsis. The Plant journal : for cell and molecular biology 36, 94-104.

Koch, K.E. (1996). Carbohydrate-Modulated Gene Expression in Plants. Annual review of plant physiology and plant molecular biology 47, 509-540.

Kwon, T., Sparks, J.A., Nakashima, J., Allen, S.N., Tang, Y., and Blancaflor, E.B. (2015). Transcriptional response of Arabidopsis seedlings during spaceflight reveals peroxidase and cell wall remodeling genes associated with root hair development. American journal of botany 102, 21-35.

Lan, P., Li, W., Lin, W.D., Santi, S., and Schmidt, W. (2013). Mapping gene activity of Arabidopsis root hairs. Genome biology 14, R67.

Landsberger, M., Lorkovic, Z.J., and Oelmuller, R. (2002). Molecular characterization of nucleus-localized RNA-binding proteins from higher plants. Plant molecular biology 48, 413-421.

Li, P., Chang, T., Chang, S., Ouyang, X., Qu, M., Song, Q., Xiao, L., Xia, S., Deng, Q., and Zhu, X.G. (2018). Systems model-guided rice yield improvements based on genes controlling source, sink, and flow. J Integr Plant Biol 60, 1154-1180.

Lu, C.A., Lim, E.K., and Yu, S.M. (1998). Sugar response sequence in the promoter of a rice alpha-amylase gene serves as a transcriptional enhancer. The Journal of biological chemistry $273,10120-10131$.

Merret, R., Descombin, J., Juan, Y.T., Favory, J.J., Carpentier, M.C., Chaparro, C., Charng, Y.Y., Deragon, J.M., and Bousquet-Antonelli, C. (2013). XRN4 and LARP1 are required for a heat-triggered mRNA decay pathway involved in plant acclimation and survival during thermal stress. Cell reports 5, 1279-1293.

Mitchell, P., and Tollervey, D. (2000). mRNA stability in eukaryotes. Current opinion in genetics \& development 10, 193-198.

Nagarajan, V.K., Kukulich, P.M., von Hagel, B., and Green, P.J. (2019). RNA degradomes reveal substrates and importance for dark and nitrogen stress responses of Arabidopsis XRN4. Nucleic acids research 47, 9216-9230. 
Nguyen, A.H., Matsui, A., Tanaka, M., Mizunashi, K., Nakaminami, K., Hayashi, M., lida, K., Toyoda, T., Nguyen, D.V., and Seki, M. (2015). Loss of Arabidopsis 5'-3' Exoribonuclease AtXRN4 Function Enhances Heat Stress Tolerance of Plants Subjected to Severe Heat Stress. Plant \& cell physiology 56, 1762-1772.

Olmedo, G., Guo, H., Gregory, B.D., Nourizadeh, S.D., Aguilar-Henonin, L., Li, H., An, F., Guzman, P., and Ecker, J.R. (2006). ETHYLENE-INSENSITIVE5 encodes a 5'-->3' exoribonuclease required for regulation of the EIN3-targeting F-box proteins EBF1/2. Proceedings of the National Academy of Sciences of the United States of America 103, 13286-13293.

Paul, M.J., and Pellny, T.K. (2003). Carbon metabolite feedback regulation of leaf photosynthesis and development. Journal of experimental botany 54,539-547.

Petricka, J.J., Schauer, M.A., Megraw, M., Breakfield, N.W., Thompson, J.W., Georgiev, S., Soderblom, E.J., Ohler, U., Moseley, M.A., Grossniklaus, U., et al. (2012). The protein expression landscape of the Arabidopsis root. Proceedings of the National Academy of Sciences of the United States of America 109, 6811-6818.

Puno, M.R., Weick, E.-M., Das, M., and Lima, C.D. (2019). SnapShot: The RNA Exosome. Cell 179, 282282.e281.

Raggi, S., Ferrarini, A., Delledonne, M., Dunand, C., Ranocha, P., De Lorenzo, G., Cervone, F., and Ferrari, S. (2015). The Arabidopsis Class III Peroxidase AtPRX71 Negatively Regulates Growth under Physiological Conditions and in Response to Cell Wall Damage. Plant physiology 169, 2513-2525.

Rodrigues, J., Inze, D., Nelissen, H., and Saibo, N.J.M. (2019). Source-Sink Regulation in Crops under Water Deficit. Trends in plant science 24, 652-663.

Ruan, Y.L. (2014). Sucrose metabolism: gateway to diverse carbon use and sugar signaling. Annual review of plant biology $65,33-67$.

Rymarquis, L.A., Souret, F.F., and Green, P.J. (2011). Evidence that XRN4, an Arabidopsis homolog of exoribonuclease XRN1, preferentially impacts transcripts with certain sequences or in particular functional categories. Rna 17, 501-511.

Sheen, J. (1990). Metabolic repression of transcription in higher plants. The Plant cell 2, 1027-1038.

Sheen, J. (1994). Feedback control of gene expression. Photosynthesis research 39, 427-438.

Sheu, J.J., Yu, T.S., Tong, W.F., and Yu, S.M. (1996). Carbohydrate starvation stimulates differential expression of rice alpha-amylase genes that is modulated through complicated transcriptional and posttranscriptional processes. The Journal of biological chemistry 271, 26998-27004.

Shikata, M., Koyama, T., Mitsuda, N., and Ohme-Takagi, M. (2009). Arabidopsis SBP-box genes SPL10, SPL11 and SPL2 control morphological change in association with shoot maturation in the reproductive 
phase. Plant \& cell physiology 50, 2133-2145.

Shishkova, S., Rost, T.L., and Dubrovsky, J.G. (2008). Determinate root growth and meristem maintenance in angiosperms. Annals of botany 101, 319-340.

Smith, M.R., Rao, I.M., and Merchant, A. (2018). Source-Sink Relationships in Crop Plants and Their Influence on Yield Development and Nutritional Quality. Frontiers in plant science 9, 1889.

Song, M.G., Bail, S., and Kiledjian, M. (2013). Multiple Nudix family proteins possess mRNA decapping activity. Rna 19, 390-399.

Souret, F.F., Kastenmayer, J.P., and Green, P.J. (2004). AtXRN4 degrades mRNA in Arabidopsis and its substrates include selected miRNA targets. Molecular cell 15, 173-183.

Tang, X., Wang, D., Liu, Y., Lu, M., Zhuang, Y., Xie, Z., Wang, C., Wang, S., Kong, Y., Chai, G., et al. (2020). Dual regulation of xylem formation by an auxin-mediated PaC3H17-PaMYB199 module in Populus. The New phytologist 225, 1545-1561.

Thomas, B.R., and Rodriguez, R.L. (1994). Metabolite Signals Regulate Gene Expression and Source/Sink Relations in Cereal Seedlings. Plant physiology 106, 1235-1239.

Vercruyssen, L., Verkest, A., Gonzalez, N., Heyndrickx, K.S., Eeckhout, D., Han, S.K., Jegu, T., Archacki, R., Van Leene, J., Andriankaja, M., et al. (2014). ANGUSTIFOLIA3 binds to SWI/SNF chromatin remodeling complexes to regulate transcription during Arabidopsis leaf development. The Plant cell 26, 210-229.

Wang, Z., Li, N., Jiang, S., Gonzalez, N., Huang, X., Wang, Y., Inze, D., and Li, Y. (2016). SCF(SAP) controls organ size by targeting PPD proteins for degradation in Arabidopsis thaliana. Nature communications 7 , 11192.

Wawer, I., Golisz, A., Sulkowska, A., Kawa, D., Kulik, A., and Kufel, J. (2018). mRNA Decapping and 5'-3' Decay Contribute to the Regulation of ABA Signaling in Arabidopsis thaliana. Frontiers in plant science 9 , 312.

Xu, J., and Chua, N.H. (2011). Processing bodies and plant development. Current opinion in plant biology 14, 88-93.

Xu, J., Yang, J.Y., Niu, Q.W., and Chua, N.H. (2006). Arabidopsis DCP2, DCP1, and VARICOSE form a decapping complex required for postembryonic development. The Plant cell 18, 3386-3398.

Yu, S.M. (1999). Cellular and genetic responses of plants to sugar starvation. Plant physiology $121,687-$ 693.

Zhang, Y., Feng, Y., Hu, Z., Hu, X., Yuan, C.X., Fan, Y., and Zhang, L. (2016). Characterization of Long Noncoding RNA-Associated Proteins by RNA-Immunoprecipitation. Methods in molecular biology 1402, 
$19-26$.

Zhang, Z.J., Gao, Q., Fang, X.D., Ding, Z.H., Gao, D.M., Xu, W.Y., Cao, Q., Qiao, J.H., Yang, Y.Z., Han, C., et al. (2020). CCR4, a RNA decay factor, is hijacked by a plant cytorhabdovirus phosphoprotein to facilitate virus replication. eLife 9 .

\section{Declarations}

Acknowledgements This work was supported by Shandong Provincial Natural Science Foundation, China (no. ZR2015CM018), the China National Transgenic Plant Research and Commercialization Project (no. 2016ZX08001-002), and the Major Program of Shandong Province Natural Science Foundation (no. ZR2018ZB0212). We thank Dr. C. Li (Institute of Genetics and Developmental Biology, Chinese Academy of Sciences) for providing the WOX5::GFP Arabidopsis line and Dr. Michael M. Goodin (University of Kentucky, USA) for providing 35S::RFP-H2B N. benthamiana line.

Author Contributions H.L. and R.Y. conceived the project. R.Y. performed most of the experiments. H.Z. carried out phenotypic analysis experiments. X.G. and H.L. conducted bioinformatics analysis. R.Y., L.G., C.Z., C.W., L.G., J.D., H.M., Y.S., S.Z. analysed data. R.Y. and H.L. wrote the manuscript.

Competing Interests Statement The authors declare that they have no competing interests.

Correspondence and requests for materials should be addressed to H.L.

\section{Figures}




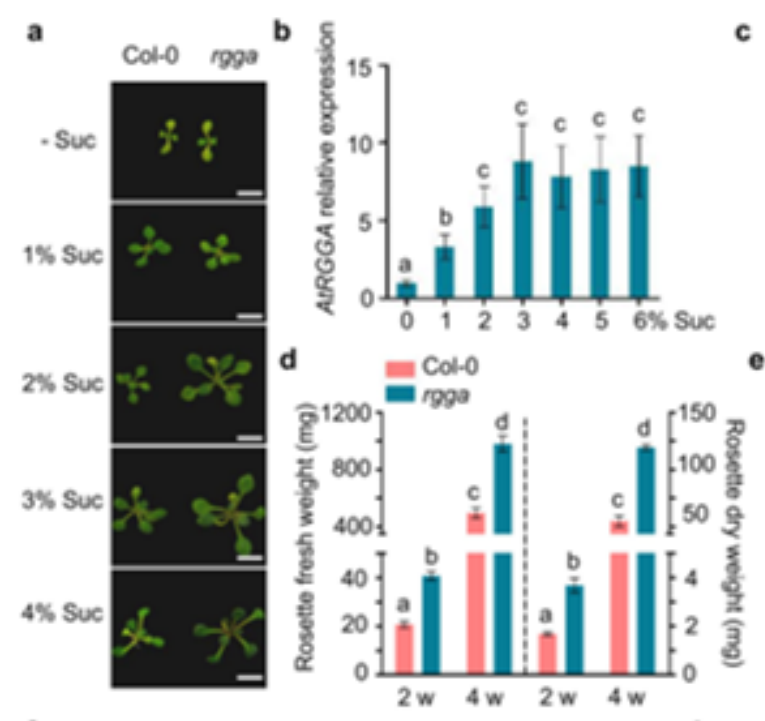

c
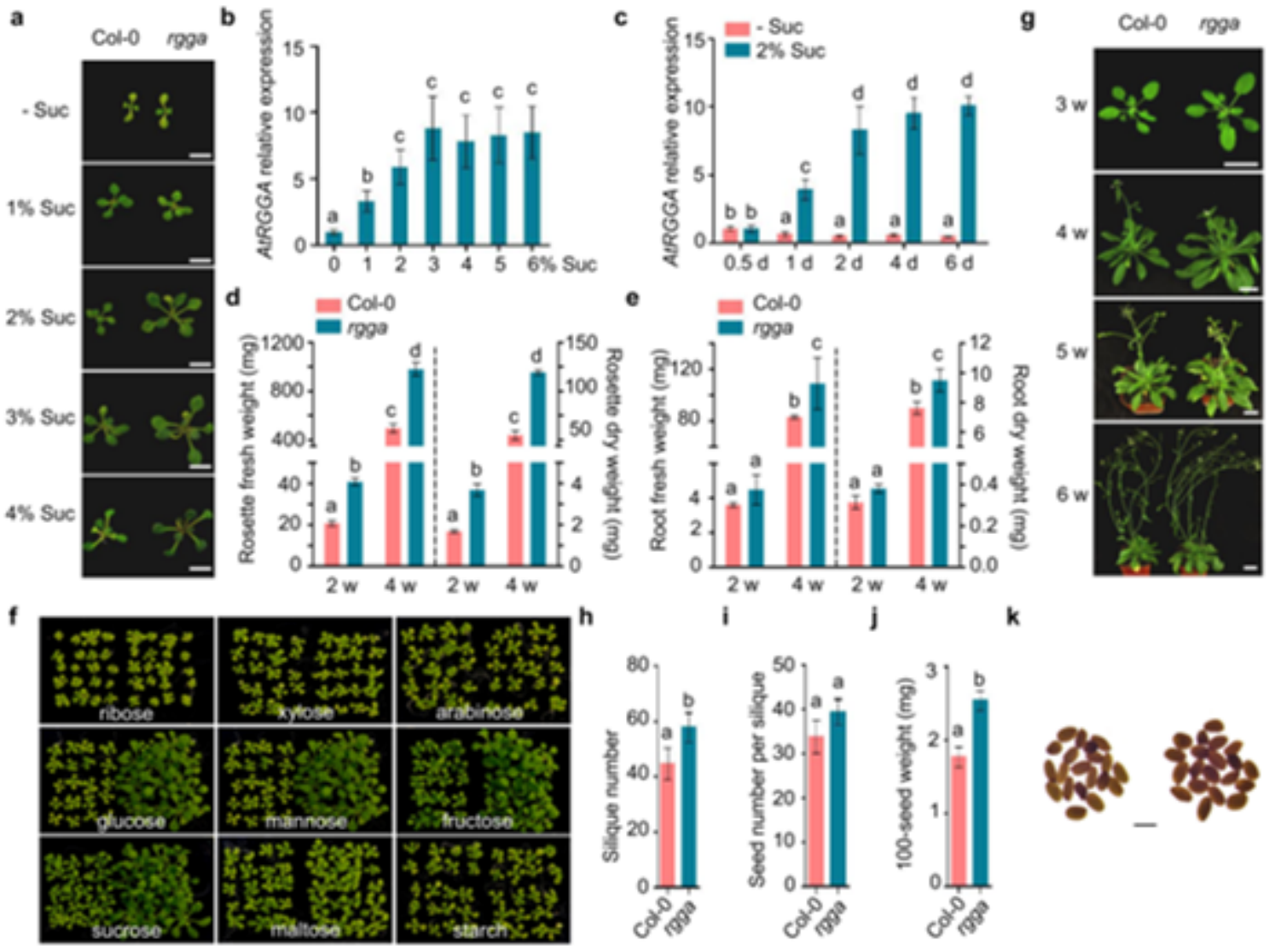

\section{Figure 1}

rgga seedlings grow faster than Col-0 when supplied with exogenous sucrose. a, Phenotypes of Col-0 and rgga seedlings grown in MS media with sucrose $(1 \%, 2 \%, 3 \%$, or $4 \%)$ for 2 weeks. Scale bar, $1 \mathrm{~cm}$. Suc, sucrose; - Suc, no sucrose. b, qPCR-based analysis of AtRGGA expression in Col-0 seedlings cultured in MS with different sucrose concentrations for 2 weeks. The AtRGGA expression level for the - Suc treatment was set to a value of 1.0. c, AtRGGA expression analysis in Col-0 seedlings cultured on $2 \%$ sucrose MS media for the indicated times; the level detected at 0.5 days was set as 1.0. One-Way Anova, $\mathrm{P}<0.05, \mathrm{n}=3$ biological replicates. $\mathrm{d}$-e, Biomass analysis of Col- 0 and rgga seedlings grown on $2 \%$ sucrose MS for $2 \mathrm{w}$ and the seedlings grown on $2 \%$ sucrose MS for $2 \mathrm{w}$ and then transferred to vermiculite for another $2 \mathrm{w}(\mathrm{n}=50)$. d, rosette fresh weight and dry weight values; e, root fresh weight and dry weight values. $f$, Growth comparison for Col- 0 and rgga seedlings grown on MS medium containing $2 \%$ ribose, xylose, arabinose, glucose, mannose, fructose, sucrose, maltose, or starch for 14 days. g-k, Rapid growth phenotypes were evident throughout the life cycle of rgga plants. Col-0 and rgga plants were cultured on $2 \%$ sucrose MS media for $2 \mathrm{w}$, and then transferred to vermiculite and grown until they produced seeds. Images of Col-0 and rgga plants at their indicated seedling ages (scale bar, $1 \mathrm{~cm})(\mathrm{g})$. Silique number per plant (h), seed number per silique (i), 100-seed weight (j), seed size (scale bar, $500 \mu \mathrm{m})(\mathrm{k})$ of Col-0 and rgga. 

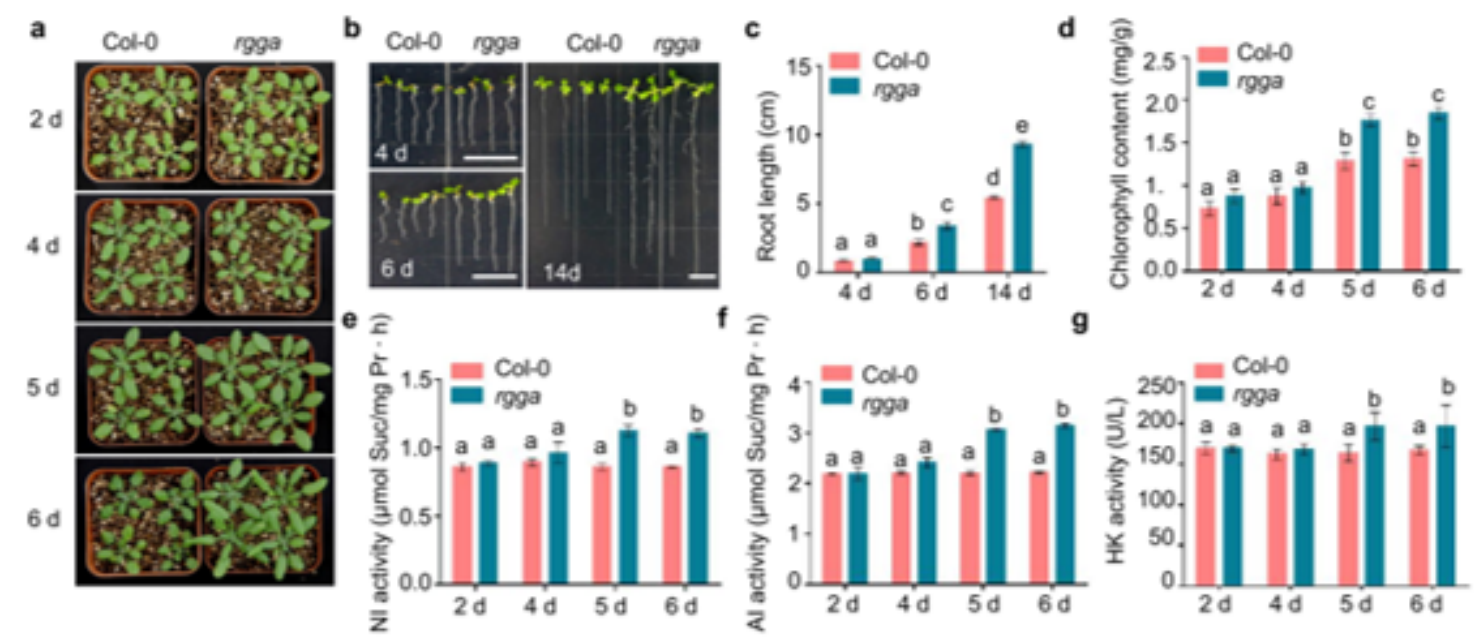

Figure 2

Exogenous sucrose supply could promote sugar metabolism, chlorophyll synthesis, and root development of rgga. Growth phenotypes (a), root images (b), primary root length determination (c), chlorophyll content (d), activity of $\mathrm{NI}$ (neutral invertase) (e), Al (acid invertase) (f), and HK (hexokinase) (g) of Col-0 and rgga seedlings which were cultured on $2 \%$ sucrose MS media for 2, 4, 5, or $6 \mathrm{~d}$, and then transferred to vermiculite until phenotypic assessment at the $4 \mathrm{w}$. One-Way Anova, $\mathrm{P}<0.05, \mathrm{n}=3$ biological replicates. Scale bar, $1 \mathrm{~cm}$. Each replicate including 50 plants. 

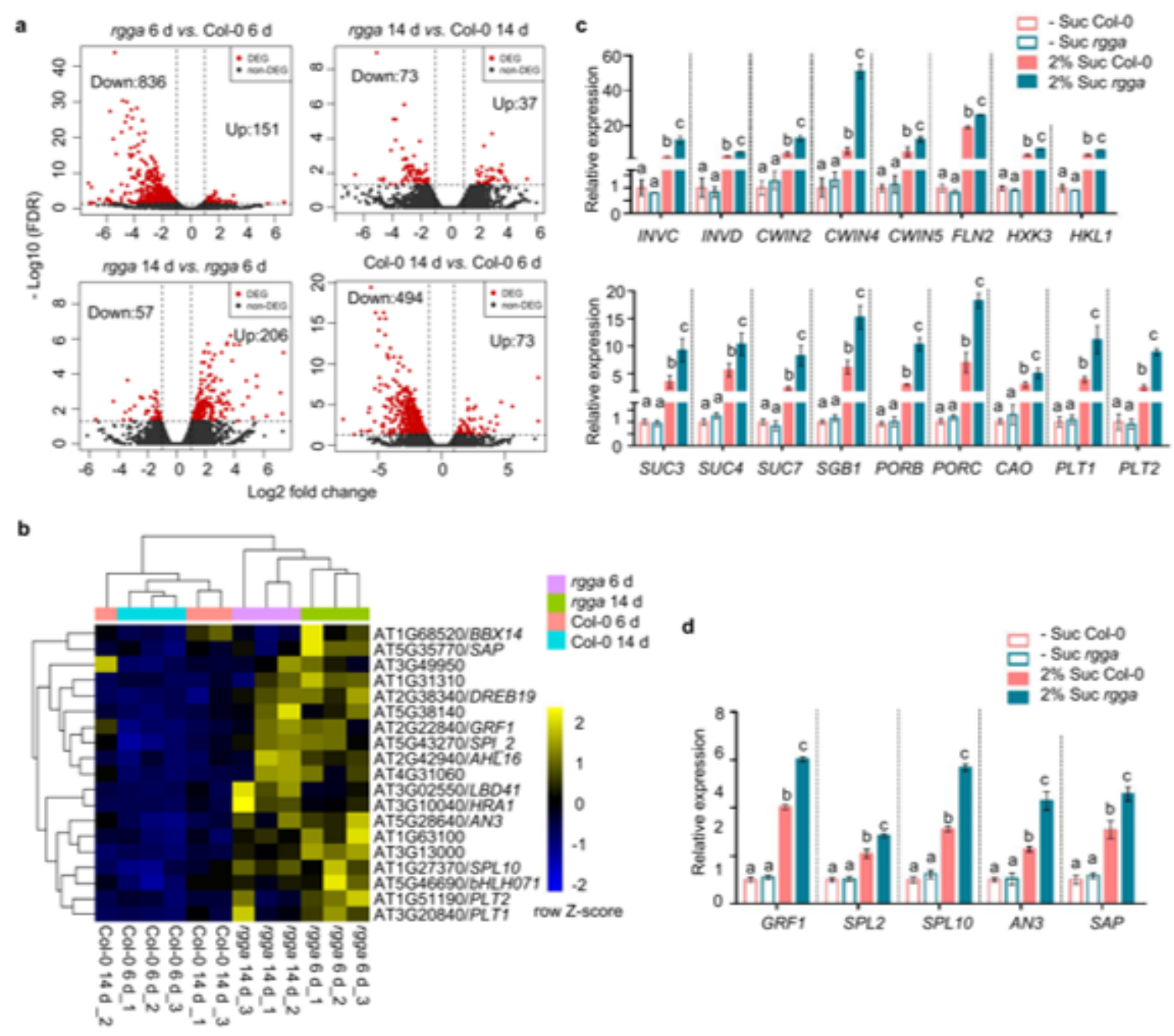

\section{Figure 3}

Transcriptome analysis of the differential expression of growth-related genes. a, Volcano plot of differential gene expression in Col- 0 and rgga seedlings cultured in $2 \%$ sucrose MS for $6 \mathrm{~d}$ or $14 \mathrm{~d}$. FDR, false discovery rate; DEG, differentially expressed genes. b, 19 transcription factor genes, including PLT1 and PLT2, were up-regulated in rgga compared with Col-0. Col-0 and rgga seedlings cultured on $2 \%$ sucrose MS for $6 \mathrm{~d}$ or $14 \mathrm{~d}$. c, Gene expression analysis by qPCR for genes encoding neutral invertase, acid invertase, hexokinase, and sucrose transporters, as well as chlorophyll synthesis and root development related genes in Col-0 and rgga seedlings cultured in sucrose free or $2 \%$ sucrose MS for 14 days. One-Way Anova, $P<0.05, n=3$ biological replicates. Suc, sucrose; - Suc, no sucrose. $d$, Expression verification of up-regulated transcription factor genes which were related to growth. rgga and Col-0 cultured in sucrose free and $2 \%$ sucrose MS for 14 days respectively. One-Way Anova, $P<0.05, n=3$ biological replicates. Suc, sucrose; - Suc, no sucrose. 

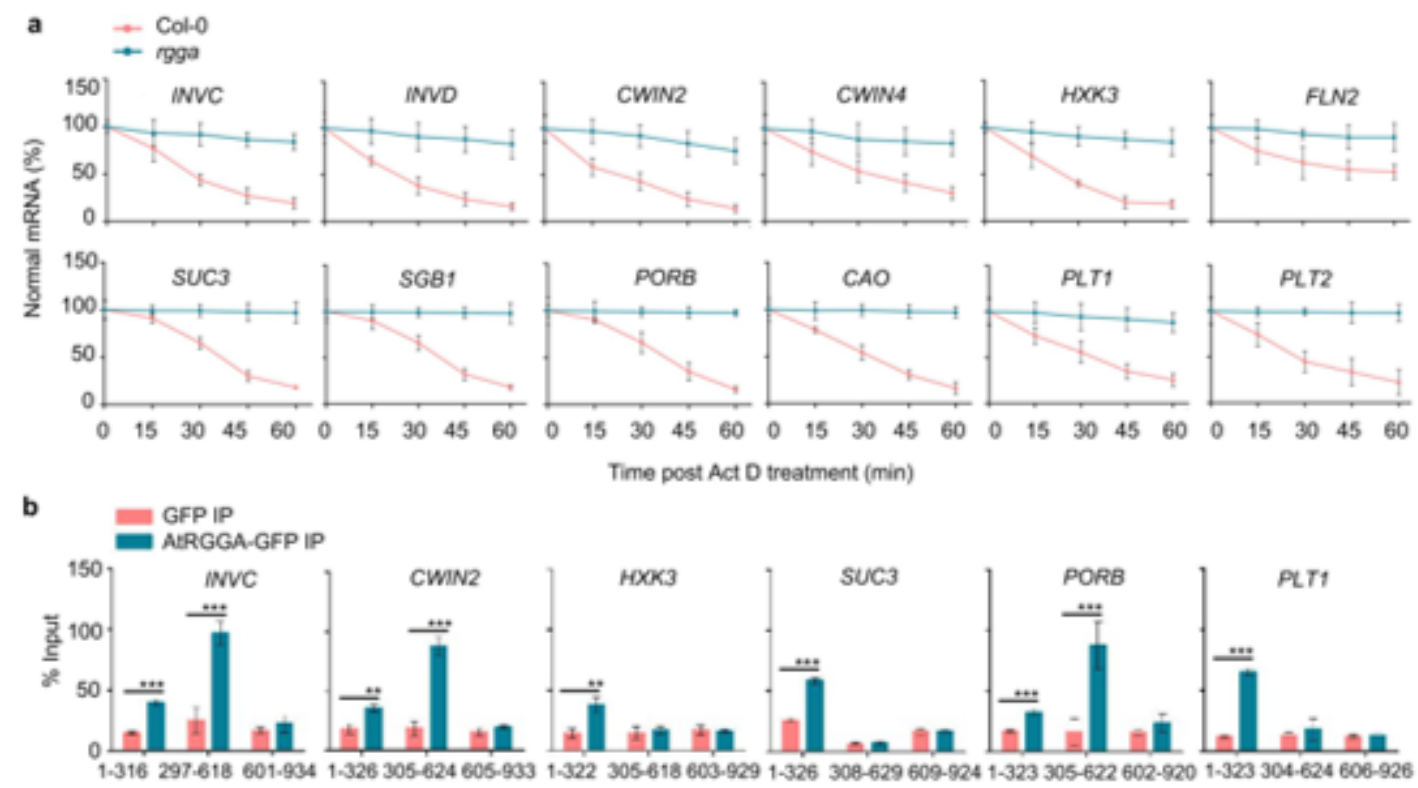

c
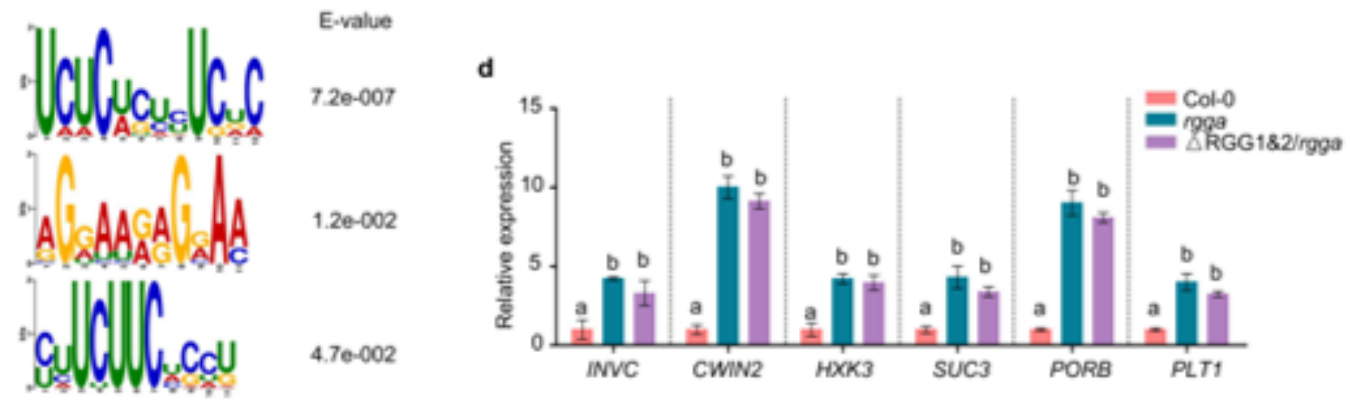

\section{Figure 4}

AtRGGA binds to 5 ' end of target mRNAs and thus affects their stability. a, mRNA stability analysis of certain genes related to sucrose metabolism and transport, chlorophyll synthesis and root development in Col- 0 and rgga. Seedlings grown in $2 \%$ sucrose MS for 14 days were treated with Act $D$ and then sampled for qPCR analysis at 15, 30, 45, and 60 min post ActD treatment. min, minutes. b, Binding region analysis of AtRGGA target mRNAs by using RIP combined with qPCR. GFP transgenic and AtRGGA-GFP transgenic Arabidopsis seedlings cultured in $2 \%$ sucrose MS for $14 \mathrm{~d}$ were sampled. Each detected mRNA was divided to several segments each about $300 \mathrm{bp}$ to detected by qPCR. Only the results of $5^{\prime}$ terminus of each target mRNA were showed here, and other results were showed in Fig. S10. One-Way Anova, $\mathrm{P}<0.05$, $\mathrm{n}=3$ biological replicates. RIP, RNA immunoprecipitation. c, Common motifs identified in the 5 terminus of the transcripts using MEME (Version 5.1.1). Analysis was performed on subsets of 6 transcripts (INVC 1618, CWIN2 1-624, HXK3 1-322, SUC3 1-326, PORB 1-622, PLT1 1-323) of which AtRGGA binding regions were verified by RIP-qPCR and 10 transcripts (1-800) of which stability increased in rgga. d, Expression levels of INVC, CWIN2, HXK3, SUC3, PORB and PLT1 in Col-0, rgga and $\triangle R G G 1 \& 2 /$ rgga plants. One-Way Anova, $\mathrm{P}<0.05, \mathrm{n}=3$ biological replicates. 


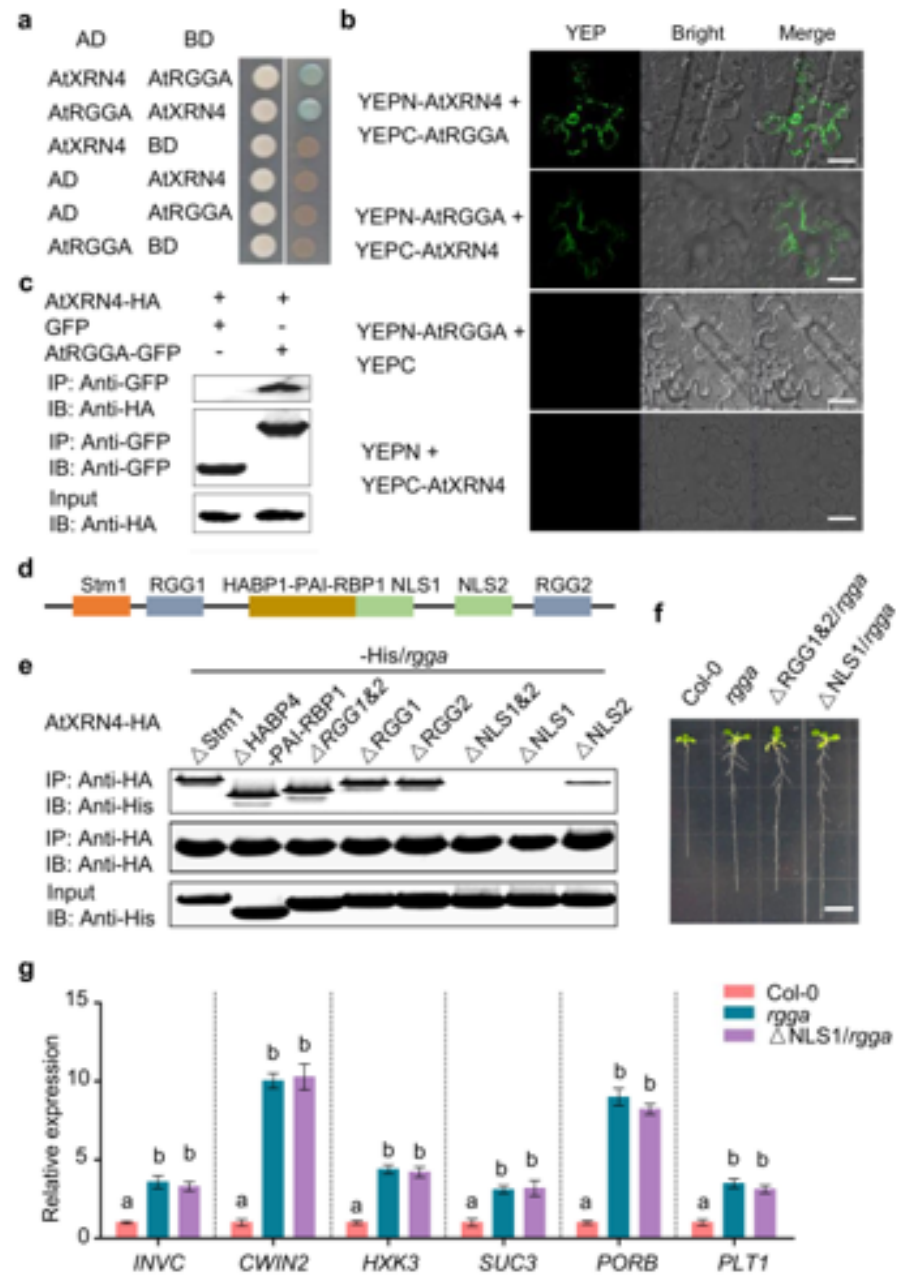

\section{Figure 5}

AtRGGA interacts with AtXRN4. a, Yeast two-hybrid (Y2H) assay of AtXRN4 and AtRGGA. Transformed yeasts were spotted on control medium (DDO) or selective medium (QDO). Empty vector transformed yeasts were used as controls. BD, DNA binding domain vector; $A D$, activation domain vector. $b$, Bimolecular fluorescence complementation (BiFC) assays of AtXRN4 and AtRGGA. Co-infiltrated leaves of N. benthamiana with YEPN-AtXRN4/YEPC-AtRGGA and YEPN-AtRGGA/YEPC-AtXRN4 at 48 hours post infiltration (hpi) were sampled. Empty vectors were used as negative controls. Scale bar, $20 \mu \mathrm{m}$. c, Coimmunoprecipitation (Co-IP) assays of AtXRN4 and AtRGGA. AtXRN4-HA was co-infiltrated with AtRGGAGFP or GFP in N. benthamiana leaves. Then the infiltrated leaves were sampled at $48 \mathrm{hpi}$ for Co-IP assay. IP, immunoprecipitation; IB, immunoblotting. $d$, The linear diagram represents the domain structure of AtRGGA protein. Stm1, Stm1 N-terminal domain; HABP4-PAl-RBP1, hyaluronan/mRNA-binding domain; RGG, arginine glycine glycine box; NLS, supposed nuclear localization signal. e, Key domain analysis of AtRGGA interacted with AtXRN4 by using Co-IP. The N. benthamiana leaves for Co-IP assay were coinfiltrated AtXRN4-HA with AtRGGA, $\triangle$ Stm1-His, $\triangle$ RBP-His, $\triangle$ RGG1-His, $\triangle$ RGG2-His, $\triangle$ RGG1\&2-His, $\triangle$ NLS1-His, $\triangle$ NLS2-His and $\triangle N$ NS1\&2-His, respectively. f, Comparison of $\triangle R G G 1 \& 2, \triangle N L S 1$ lines with Col-0 and rgga. These Arabidopsis lines were vertically cultured in $2 \%$ sucrose MS for $14 \mathrm{~d}$. Scale bar, 1 
cm. g, Expression levels of INVC, CWIN2, HXK3, SUC3, PORB and PLT1 in Col-0, rgga and $\triangle$ NLS1/rgga lines. One-Way Anova, $\mathrm{P}<0.05, \mathrm{n}=3$ biological replicates.

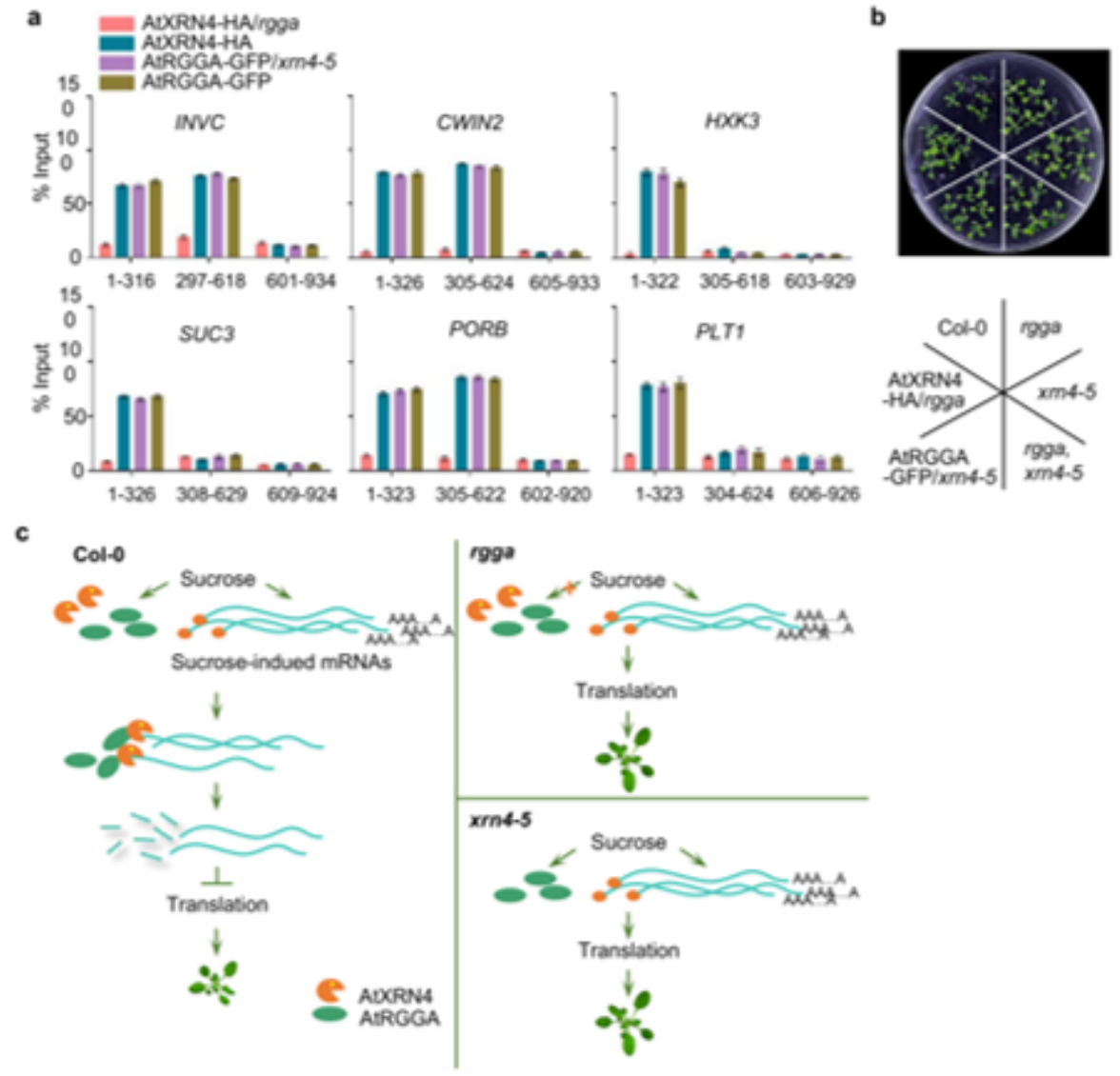

Figure 6

Sucrose promotes the growth of xrn4-5. a, AtRGGA addresses AtXRN4 to recognize the target mRNAs. The seedlings of AtXRN4-HA/rgga, AtRGGA-GFP/xrn4-5, AtXRN4-HA and AtRGGA-GFP grown in 2\% sucrose MS for $14 \mathrm{~d}$ were sampled for binding region analysis for AtRGGA targets including INVC, CWIN2, HXK3, SUC3, PORB and PLT1 mRNAs using RIP combined with qPCR. The enrichment of mRNA fragments was detected by the primer pairs each covering about 300bp. Only the results of $5^{\prime}$ end of each target mRNA were showed here, and other results were showed in Fig. S13. One-Way Anova, $P<0.05, n=3$ biological replicates. RIP, RNA immunoprecipitation assay. b, Comparison of Col-0, rgga, xrn4-5, XRN4HA/rgga, RGGA-GFP/xrn4-5 and rgga,xrn4-5 lines which were grown in 2\% sucrose MS for 14 days. c, Proposed model for the regulation mechanisms of AtRGGA on Arabidopsis growth. AtRGGA interacts with AtXRN4, and guides the degradation of mRNA subsets which related to sucrose transport and metabolism, chlorophyll synthesis, and root development by recognizing the common motif(s) located in the 5'UTRs and 5'UTR adjacent regions of mRNAs. In the absence of AtRGGA or the interaction domain, AtXRN4 itself no longer recognizes the target mRNAs, leading to a significant increase in mRNAs accumulation, thus promoting Arabidopsis growth with exogenous sucrose supplement. AtXRN4 mutant line xrn4-5 shows similar phenotypes with rgga line. The Arabidopsis images represent the relative rosette size of the seedlings grown in $2 \%$ sucrose MS for 14 days. 


\section{Supplementary Files}

This is a list of supplementary files associated with this preprint. Click to download.

- SupplementaryInformation.pdf 